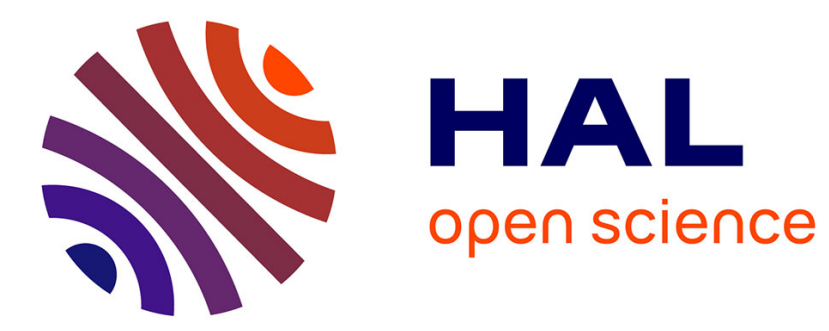

\title{
Effets de la dominance oculaire sur la coordination oeil-main dans les duels sportifs
}

\author{
G. Azemar, Jean-François Stein, Hubert Ripoll
}

\section{To cite this version:}

G. Azemar, Jean-François Stein, Hubert Ripoll. Effets de la dominance oculaire sur la coordination oeil-main dans les duels sportifs. Science \& Sports, 2008, 23 (6), pp.263-277. 10.1016/j.scispo.2008.06.004 . hal-01710264

\section{HAL Id: hal-01710264 https://hal-insep.archives-ouvertes.fr/hal-01710264}

Submitted on 15 Feb 2018

HAL is a multi-disciplinary open access archive for the deposit and dissemination of scientific research documents, whether they are published or not. The documents may come from teaching and research institutions in France or abroad, or from public or private research centers.
L'archive ouverte pluridisciplinaire HAL, est destinée au dépôt et à la diffusion de documents scientifiques de niveau recherche, publiés ou non, émanant des établissements d'enseignement et de recherche français ou étrangers, des laboratoires publics ou privés. 


\title{
Effets de la dominance oculaire sur la coordination oil-main dans les duels sportifs
}

\author{
Effects of ocular dominance on eye-hand coordination in sporting duels
}

\author{
G. Azémar $^{{ }^{a}}{ }^{\text {, J.-F. Stein }}{ }^{\text {b }}$, H. Ripoll ${ }^{\text {c }}$ \\ a 72, rond-point du Pont-de-Sèvres, 92100 Boulogne-Billancourt, France \\ ${ }^{\mathrm{b}}$ Insep, 11, avenue du Tremblay, 75012 Paris, France \\ c Laboratoire LSIS, UMR CNRS 6168, université de la Méditerranée, 58, boulevard \\ Charles-Livon, 13284 Marseille cedex 07, France \\ *Auteur correspondant. Adresse e-mail : gazem@wanadoo.fr (G. Azémar).
}

Article paru dans : Science \& Sports (ISSN : 0765-1597), 2008, vol. 23, n 6, pp. 263-277

(doi:10.1016/j.scispo.2008.06.004)

\section{Résumé}

Les duels sportifs tels que l'escrime, la boxe et le tennis de table comme certains face-à-face individuels en sport collectif, donnent lieu à des interactions visuo-motrices extrêmement rapides. Ces situations, caractérisées par une forte incertitude spatio-temporelle, exigent des protagonistes un très haut niveau d'attention visuelle. Des enquêtes réalisées dans ces sports révèlent, au plus haut niveau des classements mondiaux, une fréquence exceptionnelle des gauchers manuels, la plupart d'entre eux ayant une dominance oculaire droite. Les droitiers ayant l'œil gauche dominant s'y trouvent aussi à un taux relativement élevé. Des tâches expérimentales avec incertitude spatio-temporelle ont déjà permis de confirmer un avantage visuo-moteur, en temps de réponse, chez des sujets ayant une latéralité œil-main croisée.

Objectifs. - Le but de l'étude est d'identifier le processus central permettant d'expliquer un tel avantage. Il s'agit pour cela, d'une part, d'actualiser les données sur la dominance oculaire, d'autre part, de faire un bilan des travaux capables d'éclairer la problématique révélée par les duels sportifs.

Données actuelles. - Il apparaît que la fonction de l'œil dominant emprunte la voie géniculostriée, c'est-à-dire une voie ipsilatérale entre l'œil et le cortex hémisphérique. Par ailleurs, il se confirme que les effets spécifiques de la dominance oculaire sur la coordination œil-main n'apparaissent qu'à partir d'un certain niveau d'incertitude spatiale.

Conclusion. - Dans les conditions de tâche qui caractérisent les duels sportifs, l'œil dominant est sollicité et mis en rapport fonctionnel, via le noyau géniculé-latéral, avec l'hémisphère ipsilatéral. Pour la réponse manuelle, au contraire, la main de réponse est en rapport, via son aire motrice, avec l'hémisphère contralatéral. En conséquence, la liaison fonctionnelle entre l'entrée visuelle et la sortie motrice n'implique qu'un hémisphère chez les sujets dont l'œil dominant et la main de réponse sont eux-mêmes contralatéraux. Ces sujets font ainsi l'économie d'un transfert interhémisphérique, relativement coûteux en temps. Il en résulte pour eux un avantage, en temps de réponse, par rapport aux sujets dont l'œil dominant et la main de réponse sont ipsilatéraux.

Mots clés : Eil dominant ; Gauchers ; Incertitude spatiale ; Coordination œil-main ; Noyau géniculé latéral 


\section{Abstract}

Duel sports, such as fencing, boxing and table-tennis, like some "face-to-face" individuals in team sports, give rise to extremely fast visuo-motor interactions. These situations, characterized by a high level of uncertainty in space and time, require a very high level of visual attention from protagonists. Investigations carried out in these sports reveal, at the highest level of world ranking, an over-representation of manual left-handers, the majority of them having a right ocular dominance. Right-handers having a left dominant eye are also represented at a relatively highrate. Experimental tasks with spatio-temporal uncertainty implemented in the laboratory have made it possible to confirm a visuo-motor advantage, in response time, in subjects having an eyehand crossed laterality.

Objectives. - The purpose of this study was to identify the central process that would explain such an advantage. In order to identify such a central process, the study will, on one hand, update the data on ocular dominance, and on the other hand, it will review and evaluate a list of works able to clarify the core problem revealed by the sporting duels.

Current data. - It appears that the function of the dominant eye uses the geniculo-striated pathway, i.e., a pathway ipsilateral between the eye and the hemispheric cortex. In addition, it was confirmed that the specific effects of ocular dominance on eye-hand coordination only appears at a certain level of space uncertainty.

Conclusion. - In task conditions characterizing duel sports, the dominant eye is requested and functionally connected via the lateral geniculate nucleus to the ipsilateral hemisphere. Concerning manual responses, on the contrary, the responding hand is connected via its motor area with the contralateral hemisphere. Consequently, the functional connection between visual input and motor output involves only one hemisphere for subjects presenting a contralateral relationship between the dominant eye and the responding hand. These subjects, therefore, do not need such an interhemispheric transfer, relatively expensive in time. The result for them is an advantage in response times, compared to subjects with ipsilateral dominant eye and the responding hand.

Keywords: Dominant eye; Left-handers; Spatial uncertainty; Eye-hand coordination; Lateral geniculate nucleus

\section{Introduction}

Le sport nous donne l'occasion de découvrir, au plus haut niveau des compétitions, certaines manifestations rares de l'habileté sensorimotrice individuelle. On peut y observer une capacité spécifique de l'homme : son potentiel d'attention et d'ajustement très rapide du geste dans des situations chargées d'imprévus. Cette perspective appartient pleinement aux sports d'opposition. Ils font intervenir une multitude d'informations que les protagonistes doivent traiter en un minimum de temps pour pouvoir agir et réagir pertinemment. Les affrontements entre deux équipes de sport collectif donnent lieu à des interactions conditionnées, pour chaque participant, par des vagues de mouvements combinés dans le champ de jeu. Les multiples mobiles appartenant au jeu nécessitent une vision globale de leurs rapports dynamiques, en fonction de certains repères fixes du champ d'action.

D'autres exemples d'une telle problématique sont apportés par les sports où s'affrontent seulement deux adversaires face-à-face. Ces duels, aussi fertiles en aléas que les sports d'équipes, se prêtent mieux à des analyses neuroscientifiques. Tous ces affrontements sportifs passent par des situations où intervient un degré extrême d'incertitude, à la fois spatiale, temporelle et événementielle. Et ce sont essentiellement des signaux visuels qui vont permettre aux participants de réduire, le plus vite et le mieux possible, cette incertitude. Il en découle une sollicitation 
maximale du système nerveux central des protagonistes afin de contrôler en permanence, et souvent dans l'urgence, les rapports entre perception et action.

Nous prendrons ici comme cas d'étude les sports d'opposition individuels où les protagonistes ne se trouvent pas a priori en corps à corps, comme la lutte ou le judo, mais à distance relativement limitée, comme l'escrime, la boxe et le tennis de table. Il existe aussi des duels comparables en sport collectif lorsque deux joueurs sont en concurrence face-à-face pour la maîtrise du ballon. Ces oppositions individuelles ont deux caractéristiques communes. D'une part, elles font intervenir en priorité les perceptions visuelles qui permettent - au contraire de la proprioception de saisir les signaux à distance et ainsi de se préparer à y répondre, voire d'anticiper l'action que prépare l'adversaire. D'autre part, la courte distance entre les protagonistes limite leur temps de décision pour le choix des actions. La durée des trajectoires des mobiles maniés par les adversaires étant souvent inférieure à la demi seconde, leur interception exige des temps de réaction très courts.

Dans ces conditions, l'issue des échanges entre les protagonistes dépend de leur aptitude respective à maîtriser des coordinations entre les entrées perceptives visuelles et les sorties motrices s'exprimant, en général, au niveau de la main. Il est vrai qu'en football, le pied qui agit sur la balle se trouve soumis à des conditions relativement comparables. Par ailleurs, chacun des acteurs doit entretenir à l'égard de son adversaire direct le maximum d'incertitude sur ses propres intentions. C'est par ses propres initiatives dans ce domaine qu'il peut gagner des millisecondes supplémentaires pour prendre l'avantage lors d'une interaction.

Des observations effectuées au plus haut niveau des compétitions d'escrime, de tennis de table et de quelques autres disciplines apparentées ont révélé l'émergence statistique de certaines caractéristiques individuelles, plus largement représentées en tête des classements. Ce fait est reconnu depuis longtemps pour ce qui relève de prédispositions physiques ou morphologiques (ex. : une grande taille en basket, une morphologie appropriée en gymnastique, des capacités cardiorespiratoires ou musculaires adéquates en athlétisme, etc.). C'est un constat d'un autre ordre qui a pu être fait en sport d'opposition. Il renvoie plutôt à la neuropsychologie et relève souvent des asymétries fonctionnelles. Des recherches dans ce domaine ont commencé durant les années 1980. En tir à la carabine, les droitiers francs se montraient les meilleurs [1]. En escrime, ce fut au tour des gauchers de s'illustrer [2]. Peu après, une sur-représentation des gauchers manuels dans l'élite sportive s'est aussi révélée significative en tennis de table, en boxe et, à un moindre degré, en tennis.

Deux autres constats singuliers étaient bientôt faits en escrime à propos des gauchers manuels. D'une part, presque tous avaient une dominance oculaire droite, c'est-à-dire qu'ils avaient une latéralité croisée entre l'œil et la main. D'autre part, ces gauchers s'illustraient seulement - mais de façon hautement significative - à l'épée et au fleuret, qui sont des armes de pointe dites " d'estoc ", dont seule l'extrémité distale permet des touches valides. Au contraire, les droitiers dominaient toujours largement au sabre, arme « d'estoc et de taille » qui permet de toucher tant avec la pointe qu'avec les faces latérales de l'arme. Ces différences ont été analysées et interprétées par ailleurs [3].

Le relatif avantage revenant à l'usage de la main gauche dans ces sports $^{1}$ a trouvé des explications pertinentes durant les années 1990 sur des bases expérimentales. Plusieurs travaux ont confirmé cet avantage, par rapport à la main droite, en temps de réaction, dans des tâches faisant intervenir des conditions d'incertitude spatio-temporelle [4]. L'interprétation générale de cette donnée s'appuie sur le rapport privilégié de la main gauche avec l'hémisphère droit, spécialisé dans la représentation centrale de l'espace visuel [5]. En cas de projection de la main gauche vers une cible, cet avantage semble s'exprimer, grâce au " colliculus supérieur » [6], et dès le début du mouvement sous la forme d'une programmation balistique de la trajectoire de 
l'extrémité du membre (ou de la pointe de l'arme). Ce phénomène, qui renvoie à la théorie prémotrice de l'attention [7], sera détaillé plus loin. Le pouvoir de déclencher une telle impulsion, orientée d'emblée vers sa cible, semble constituer un avantage avec des armes d'estoc.

De son côté, la main droite tire de ses liens directs avec l'hémisphère gauche un autre type d'avantage qui s'exprime mieux avec un sabre : elle est plus apte à un contrôle continu de la trajectoire du membre au cours de son déroulement [8,9]. Le cortex pariétal postérieur gauche semble jouer un rôle de « pilote automatique » pour la main droite [10,11]. Il s'agit d'un véritable contrôle " en ligne ». Cela facilite à la main droite les nombreux changements de direction qu'exige un sabre, durant sa trajectoire, afin de pouvoir toucher ou frapper de diverses façons en fin de course. Les trajectoires de cette arme ont, en effet, des formes fouettées plus complexes et variables que celles d'une arme d'estoc.

Quant à la latéralité œil-main croisée signalée chez les gauchers s'illustrant aux armes d'estoc, elle a été vérifiée individuellement au moyen d'un test spécifique de la dominance oculaire, appelé hold-in-card, qui sera décrit un peu plus loin. Ces contrôles ont eu lieu à l'occasion des compétitions mondiales majeures (jeux Olympiques [JO] et championnats du Monde) [12]. Un cas extrême s'est produit lors des JO de Moscou (1980), où huit gauchers s'étaient hissés aux huit premières places du fleuret masculin. Il a été constaté après la finale que sept d'entre eux avaient une dominance oculaire droite. La même année, on comptait neuf gauchers manuels dans l'équipe de France (sur un total de 23 escrimeurs) et huit d'entre eux avaient l'œil droit dominant. Ce constat d'une forte majorité de gauchers ayant l'œil droit dominant s'est confirmé ensuite plus largement dans l'élite mondiale. Dans la population générale, comparativement, seulement deux gauchers sur dix sont dans ce cas. Une enquête portant sur 352 sportifs de haut niveau représentant 23 disciplines, dont 15 sports d'opposition, a révélé que les cas de latéralité œilmain croisée, droitiers et gauchers confondus, s'y trouvaient représentés à un taux statistiquement plus élevé $(38,6 \%)$ que dans un échantillon témoin de la population générale $(23,6 \%)$ [12].

Il reste à éclaircir le rôle que peut jouer l'œil dominant, en relation avec la main préférentielle, dans la problématique attentionnelle des sports d'opposition. Les recherches bibliographiques entreprises dans cette direction montrent que la notion même de dominance oculaire, bien que soulevée dès l'antiquité et encore rappelée au XVIe siècle [13], est demeurée largement négligée dans les travaux modernes de neurosciences. On s'y intéresse un peu plus depuis quelques années, mais c'est seulement à partir de 2005, qu'ont enfin paru des rapports de recherches permettant d'éclairer la problématique évoquée ci-dessus. Ceux-ci nous apportent des arguments en faveur d'un modèle d'interprétation des faits observés.

Avant de déboucher sur une telle synthèse explicative, il semble nécessaire d'effectuer un rapide tour d'horizon du contexte théorique, réactualisé à cette occasion. Il portera sur certains aspects fonctionnels de l'appareil visuel et du système nerveux central tels qu'ils sont sollicités dans les tâches qui viennent d'être évoquées. Des données récentes d'imagerie fonctionnelle ont renouvelé l'approche des processus mis en jeu. Il s'agira aussi d'apporter quelques éclaircissements sur la dominance oculaire, son identification, son rôle et ses supports fonctionnels.

\section{L’attention visuelle et ses systèmes dédoublés}

Il s'agit de rappeler ici sommairement certaines grandes voies, entre le cortex et le système visuel, ayant deux composantes distinctes. On ne manquera pas d'évoquer aussi, dans cette perspective, l'asymétrie introduite par les fonctions différenciées des hémisphères, à l'origine de la plupart des biais latéraux observés sur le terrain aux niveaux manuel ou podal. Seront aussi revues quelques notions trop souvent négligées comme la rivalité binoculaire et les deux 
principaux types de saccades, selon leur mode de déclenchement. Des circuits dédoublés, à différents étages, entre notre système visuel et ses centres de traitement ou de contrôle corticaux et sous-corticaux - ouvrent ainsi la voie à des fonctions couplées, antagonistes ou complémentaires.

L'homme n'étant pas un cyclope, ses deux yeux lui confèrent des avantages supplémentaires en coopérant - notamment pour une vision en $3 \mathrm{D}$-, mais au risque de se trouver parfois en rivalité. Cette « rivalité binoculaire » a fait l'objet de nombreuses études depuis longtemps. Les plus récentes ont permis de mieux décrire les bases neurales [14] et fonctionnelles $[15,16]$. Dans la vie quotidienne, une telle rivalité pourrait être une source de conflits compromettant la vivacité et la souplesse adaptative de notre vision si l'un des yeux n'avait pas obtenu un rôle dominant. Cette dominance lui permet notamment d'initier rapidement et de guider les déplacements conjugués des deux axes optiques lors des saccades oculaires [17].

L'organisation anatomique du système visuel est symétrique par rapport au plan sagittal. En outre, dans chaque œil, une autre organisation bilatérale de la rétine repose sur la distinction fonctionnelle de deux « hémirétines ». Situées de part et d'autre du plan sagittal passant par l'axe optique de l'œil, leurs fibres afférentes empruntent des chemins différents. La ligne de partage entre ces hémirétines constitue le « méridien vertical rétinien ». Deux sortes de voies conduisent, en effet, les entrées perceptives des hémirétines vers les centres de traitement (Fig. 1). L'une croise entre l'œil et l'hémisphère, l'autre non. Celle qui croise, la plus archaïque, est la voie " rétino-tectale ». Partant de l'hémirétine nasale, elle croise la ligne médiane pour rejoindre le « colliculus supérieur »- structure sous-corticale - et se projette ensuite dans le cortex visuel de « l'hémisphère contralatéral à l'œil d'origine ». Les images transportées par cette voie représentent, pour chaque œil, 90॰ de l'hémichamp visuel temporal. Ce premier sous-système contribue beaucoup à la vision périphérique qui joue un rôle déterminant pour détecter des signaux dans un champ étendu. L'autre voie, dite " rétino-géniculée », phylogénétiquement plus récente, part de l'hémirétine temporale, rejoint le « noyau géniculé latéral » (NGL) sans croiser la ligne médiane, puis se projette ainsi dans le cortex visuel primaire de « l'hémisphère ipsilatéral à l'œil d'origine ». Les images transportées par cette seconde voie couvrent seulement $60^{\circ}$ de l'hémichamp visuel nasal et privilégient la « région fovéale ». Ce sous-système contribue à l'identification de certains détails de l'image parvenue en vision centrale.

Au total, grâce à ces deux sous-systèmes, les deux yeux contribuent à la fois, mais de fac, on relativement différente, à projeter chaque hémichamp visuel dans l'hémisphère contralatéral. La représentation de l'espace doit être réalisée par les centres en mixant les images provenant des deux hémisphères. La jonction se fait via le corps calleux. Le bord médian de chaque hémichamp visuel va contribuer à la production d'un méridien vertical rétinien fusionné. La verticalité de celuici est objective quand, la tête étant stabilisée par le réflexe vestibulo-oculaire, les deux yeux sont alignés sur l'horizontale. Dans cette représentation centrale, la superposition des deux projections fovéales facilite l'ajustement des hémichamps le long d'un méridien virtuel qui représente alors, par analogie, la référence verticale de l'espace d'action. Il a été démontré, dans cette perspective, que l'hémisphère droit contribue spécifiquement à la stabilisation visuelle de la tête dans l'espace [18].

La combinaison des projections des quatre hémirétines contribue enfin à la vision stéréoscopique. Donnant un aperçu de la profondeur du champ, celle-ci rend ainsi mieux compte de la distribution topologique des objets dans l'espace extérieur. La binocularité apporte donc secondairement une troisième dimension aux projections rétiniennes. Lors d'une tâche de visée, c'est pourtant à la seule fovéa de l'œil dominant que revient le privilège d'apporter à la représentation visuelle de l'espace un point d'ancrage pour un « référentiel égocentrique », c'est- 
à-dire référé au corps [19-21]. Un codage polarisé des rapports spatiaux permet alors au sujet de mieux aligner le geste et l'action sur une cible éloignée.

L'attention en vision centrale s'appuie sur la fovéa. Cette forme d'attention est dite «manifeste » (de l'anglais overt) quand elle guide et accompagne les déplacements du regard vers divers points de fixation. Cependant, un autre type d'attention, plus largement utilisé en sport d'opposition, est l'attention dite « latente » ou parfois « masquée » (de l'anglais covert). Le colliculus supérieur y joue le premier rôle [22]. Cette sorte d'attention peut être mobilisée, sans que bougent les yeux, vers divers secteurs périphériques du champ visuel, où entre alors en jeu un " référentiel allocentrique » c'est-à-dire référé à l'espace [19,20]. Dans cet espace organisé, certains signaux aléatoires peuvent survenir à tout instant. Si le sujet était en terrain inconnu, un tel signal périphérique donnerait lieu à une saccade oculaire afin de vérifier sa teneur en vision centrale. En duel, ce signal doit avoir été pré-identifié pour être déjà reconnaissable en vision périphérique. Un entraînement spécifique permet alors d'assortir une réponse manuelle ciblée à tel signal attendu [23], de sorte qu'une projection de la main dans sa direction peut accompagner la saccade dès son départ, au lieu de la suivre [7,24]. Le gain de temps est considérable. Une étude vient de montrer qu'une fixation oculaire préalable augmenterait la latence œil-main d'au moins 120 millisecondes [25].

En relation avec la nature de l'attention, il faut aussi distinguer deux sortes de saccades. L'attention dite manifeste recourt typiquement à des "saccades volontaires » plus ou moins « ciblées », caractérisées par de longues latences. Leur production est hautement contrôlée, à partir du cortex préfrontal, grâce à des interactions du champ oculaire frontal avec le colliculus supérieur [26]. Le cortex pariétal postérieur, pour sa part, joue un rôle dans l'intégration visuospatiale des informations saisies par ce moyen [27]. Quant à l'attention dite latente, elle recourt plutôt à des « saccades express », déclenchées de façon plus automatique (à l'étage sous-cortical) par des signaux aléatoires. Ces « saccades express » ont de très courtes latences, de l'ordre de 80 à 100 millisecondes [28]. Ce sont des saccades de ce type qui peuvent être accompagnées immédiatement par une projection ciblée de la main. Il a été, en effet, démontré chez le singe que ce processus est dû à une connexion directe, dans le " colliculus supérieur ", entre la couche superficielle qui reçoit les entrées visuelles et la couche profonde d'où émergent les sorties motrices $[29,30]$. Une telle «porte » de communication est cependant modulée, selon le contexte, à partir du cortex cérébral et des ganglions de la base.

Lors des saccades, la coordination motrice entre les axes oculaires (déplacements conjugués et vergence) s'appuie sur des rapports étroits entre les champs oculaires corticaux et le colliculus supérieur. Celui-ci est aussi en interaction par ailleurs avec le cervelet par des voies cérébellotectales [31] qui permettent d'assurer la précision des réponses motrices grâce à des circuits complexes impliquant aussi bien l'équilibre postural que le contexte spatial.

Pour compléter ce rapide tour d'horizon, il faut encore évoquer quelques grands schémas fonctionnels dédoublés, attribués plus généralement aux centres supérieurs, mais qui ne manquent pas d'influencer l'attention visuelle.

Il est bien connu que les fonctions dominantes des hémisphères cérébraux sont respectivement la gestion du langage pour l'hémisphère gauche et celle de l'espace pour l'hémisphère droit. Aujourd'hui, il est devenu souhaitable de distinguer les hémisphères non plus par «ce » qu'ils gèrent, mais plutôt par " la façon » dont ils traitent les informations, éventuellement les mêmes informations [12]. Ainsi, des mécanismes attentionnels différenciés seraient à l'origine des spécialisations respectives des hémisphères [32,33]. Le " projecteur » attentionnel de l'hémisphère gauche est très focalisé, favorisant une recherche séquentielle d'informations ponctuelles. Il traite les événements que nous vivons de façon sérielle, s'attachant à leur évolution et aux causes de celle-ci. Il permet, en quelque sorte, une saisie analytique des facteurs 
de changement. Le vécu y est traité « en ligne ». Le langage certes, mais aussi la conduite de nos mouvements relèvent d'un tel processus [34,35]. Quant au «projecteur » de l'hémisphère droit, il s'étend, au contraire, sur des groupes d'items, utilisant une stratégie qui permet le traitement simultané de plusieurs sources d'information. Il traite les événements à chaque instant comme des clichés successifs, y cherchant des rapports " en arborescence » entre les éléments qui s'y trouvent. La saisie holistique de ces rapports transversaux s'effectue, pour l'essentiel, hors du champ de conscience. Cependant, comme le montre l'entraînement sportif, la richesse de ses acquisitions dépend du nombre et de la qualité des expériences actives réalisées. Et la représentation de l'espace en est l'illustration, avec les référentiels qui en résultent. Ces dominantes fonctionnelles différenciées entre les deux hémisphères se reflètent bien, contralatéralement, dans les caractéristiques respectives des membres supérieurs droit et gauche (évoquées ci-dessus, page 3, paragraphes 1 et 2).

Il faut enfin évoquer deux autres schémas dédoublés de l'attention dont la faveur varie, selon les écoles, dans les publications de neurosciences. Le premier schéma date d'une quinzaine d'années [36]. Il distingue deux sortes de voies, respectivement "ventrales » et " dorsales », pour la perception et l'action. D'une part, un « flux ventral » de projections, émanant du cortex visuel primaire à destination du cortex temporal inférieur, contribue à l'identification perceptive des objets. D'autre part, un " flux dorsal », plus rapide, conduit des opérations sensorimotrices permettant de guider les actions vers des objets, fixes ou en mouvement [37]. Ce dernier part aussi du cortex strié, mais à destination de la région pariétale postérieure. Ces deux soussystèmes, ventral et dorsal, interagissent en boucle dans le contrôle sensorimoteur des gestes de projection [38].

L'autre grand schéma, plus récent, distingue aussi deux sous-systèmes proches des précédents, mais relatifs au sens de déroulement, descendant ou ascendant, du processus attentionnel dans le système nerveux central $[39,40]$. L'un est dit top-down, littéralement de haut en bas, quand, fondé sur une instruction ou une décision - processus endogène - il s'engage à partir du cortex préfrontal. L'autre est dit, au contraire bottomup, de bas en haut, quand, suscité par un stimulus inattendu - processus exogène - il s'engage à partir du cortex pariétal postérieur, très impliqué dans l'initiation des saccades express. Remarque : à ce dernier niveau, le cortex pariétal postérieur droit (CPPd) interviendrait plutôt dans le désengagement d'une précédente fixation visuelle, tandis que le gauche $(\mathrm{CPPg})$ présiderait à la réorientation du regard [41].

Ce schéma général vient d'être validé chez le singe [42] et chez l'homme [43]. Dans ce dernier cas, c'est en IRM fonctionnelle (IRMf) que s'est vérifiée une dissociation neuroanatomique entre les deux sortes de flux chez des sujets en train d'effectuer des tâches visuo-manuelles dans des conditions variées d'incertitude spatiale. Ainsi, se sont confirmés en laboratoire deux sortes de processus que suggéraient des observations de terrain. Un processus top-down est appelé à intervenir quand la réponse au signal-cible doit tenir compte d'informations, données par un présignal, qui entretiennent l'incertitude spatiale. Ce processus donne lieu à des réponses hautement contrôlées. Au contraire, en l'absence de présignal et d'incertitude notable, c'est un processus bottom-up qui intervient. Le signal joue alors le rôle d'un stimulus exogène qui alerte le système nerveux central (SNC), suscitant une réponse plus directe et automatique dans sa direction. Et le temps de réaction varie avec le degré d'incertitude.

Bien que ces systèmes dédoublés soient a priori symétriques, au moins structurellement, la problématique des sports d'opposition va y introduire des biais latéraux déterminants. L'asymétrie fonctionnelle y dépend à la fois de la main mise en jeu et de l'œil dominant. 


\section{La dominance oculaire : objectivation et supports neuraux}

Il y a déjà un quart de siècle qu'a été apportée la preuve électrophysiologique, à partir des potentiels évoqués visuels, d'une différenciation fonctionnelle entre les deux yeux [44]. Une étude plus récente en IRMf a montré que l'œil dominant active une aire plus large que celle de l'œil non dominant dans le cortex visuel primaire [45].La dominance de l'un des yeux évoquée ainsi n'est pourtant pas d'ordre sensoriel, mais moteur ${ }^{2}$ et psychomoteur [46]. Elle rejoint en cela les manifestations de la latéralité fonctionnelle plus connues à l'étage des mains ou des pieds. Cependant, on reconnaît en plus à l'œil dominant des effets perceptifs secondaires influençant, de façon subconsciente, la représentation centrale de l'espace d'action. Il apparaîtra aussi ci-après qu'il joue un rôle important dans l'orientation des saccades.

Il faut savoir que la dominance oculaire est plus fréquemment senestre que la dominance manuelle ou podale. En effet, environ trois individus sur dix ont l'œil gauche dominant tandis que seulement un sur dix se sert préférentiellement de sa main gauche. Cela entraîne une corrélation réduite entre ces deux niveaux de latéralité individuelle. Ainsi, un quart de la population présente une latéralisation œil-main non homogène. La latéralité oculaire n'est probablement pas héréditaire. Une étude portant sur 51 paires de jumeaux, dont 31 de monozygotes, montre que l'asymétrie perceptive visuelle ne relève pas d'une transmission génétique [47].

Le plus banal des rôles de l'œil dominant se manifeste dans des tâches de visée quand s'impose le choix d'un œil, par exemple, pour regarder à travers un petit orifice en vision monoculaire. Cependant, quand le but est d'identifier l'œil dominant d'un individu, il est souhaitable de recourir à des tâches de visée pouvant être réalisées en gardant les deux yeux ouverts. Elles incitent le sujet à mettre les deux yeux en concurrence, en principe, avant de placer l'œil supposé dominant devant un repère proximal pour l'aligner ensuite sur une cible éloignée. Le choix s'y effectue de fac, on intuitive ou par essais et erreurs. Le sujet tend à adopter l'œil qui, une fois aligné sur la cible, lui permet de se sentir en meilleure condition pour se situer lui-même dans l'espace d'action. Il utilise alors cet œil comme un repère égocentrique [18,20]. Les tests fondés sur de telles tâches de visée sont cependant assez inconstants, donc peu fiables.

À propos de visée, l'œil préférentiel pose parfois des problèmes aux sportifs spécialisés en tir à l'arc ou à la carabine. Des contraintes matérielles font qu'en principe, dans ces disciplines, la main qui déclenche le tir doit se trouver du même côté que l'œil dominant. Cela suppose une latéralité œil-main homogène. Les cas de latéralité œil-main croisée créent une ambiguïté bien connue des sportifs en cause et de leurs entraîneurs. Il s'avère que la plupart d'entre eux la résolvent empiriquement en adoptant l'arme et la posture manuelle d'un gaucher homogène (œilmain) si c'est leur œil gauche qui est dominant, ou en faisant l'inverse si c'est l'œil droit. Cela tend à montrer que la fonction de l'œil prime sur celle des membres dans ce type de tâche.

L'autre rôle de l'œil dominant, impliqué dans la conjugaison des mouvements oculaires, a été et reste encore trop souvent méconnu. Il s'agit du rôle directionnel de l'un des yeux au début d'une saccade vers un signal détecté dans le champ visuel. Cet aspect est pourtant confirmé expérimentalement depuis plus de dix ans [48]. Il est vrai aussi que c'est tout récemment qu'ont été accomplis des progrès déterminants dans ce domaine. Ils sont dus à des travaux originaux dont la plupart proviennent d'équipes japonaises. Ainsi, une exploration en vidéo-oculographie chez des sujets droitiers homogènes a montré que la vitesse des saccades horizontales de l'œil dominant est significativement plus élevée que celle de l'œil non dominant [17]. Un peu plus tôt, une exploration intracellulaire des noyaux géniculés latéraux droit et gauche du chat avait montré qu'ils interviennent différentiellement dans la gestion de la rivalité binoculaire en se chargeant respectivement de l'œil dominant et de l'œil non dominant [49]. 
C'est surtout grâce à la multiplication récente de travaux consacrés à la rivalité binoculaire $[14,15]$ que se sont dégagées de nouvelles données objectives sur le rôle de l'œil dominant dans cette perspective. Comparant diverses méthodologies, c'est encore une équipe japonaise qui a précisé les conditions adéquates pour que se manifeste clairement la dominance oculaire. Les auteurs concluent leur étude en désignant l'épreuve hold-in-card ou épreuve du " trou dans la carte » comme le plus fiable des tests, à ce jour, pour identifier la dominance oculaire [50]. Dans le même but, ils ont aussi proposé un moyen d'évaluation quantitative en associant des rétinomètres à la mise en œuvre de la rivalité binoculaire [51]. Le test hold-in-card n'est pas nouveau [52], mais il faut le réaliser avec soin pour pouvoir être assuré de ses données ${ }^{3}$. Son intérêt pour tester la dominance oculaire vient d'être encore confirmé [53].

Les explorations de la rivalité binoculaire ont permis en outre de confirmer chez l'homme l'implication du noyau géniculé latéral (NGL) dans les processus mis en jeu. Ce noyau a été désigné comme "l'étape la plus précoce du traitement visuel qui reflète une dominance spécifique d'un œil »[54]. Simultanément, deux autres équipes concluaient que le NGL, classiquement vu comme le « portail » du cortex visuel, est une sorte de " garde-barrière précoce de l'attention visuelle » $[55,56]$. L'analyse des processus visuels intervenant à ce niveau montre que chaque NGL exerce une fonction différente, soit une dominance, soit une suppression, selon l'œil qu'il représente sur la voie centripète [57] (Fig. 1).

Cette série de travaux démontre que les fonctions spécifiques de l'œil dominant empruntent la voie rétino-géniculée qui achemine notamment les flux visuels de l'hémirétine temporale vers l'hémisphère ipsilatéral. Voilà une donnée déterminante pour l'interprétation générale des phénomènes observés. Une étude vient encore de confirmer le processus donnant la priorité à l'œil dominant dans le traitement de certaines informations visuelles. Lors d'une tâche d'exploration visuelle, cette priorité repose bien sur une relative inhibition des afférences centrales issues de l'œil non dominant [58].

On peut se demander quand et comment se détermine la dominance d'un œil au cours du développement individuel. L'hypothèse a déjà été formulée de son apparition durant les premiers mois de la vie, suggérée par les épisodes frustes et transitoires de microstrabisme souvent constatés de façon fugace chez les nourrissons de cet âge [12]. Ces épisodes semblent refléter une période d'adaptation de la conjugaison des mouvements oculaires. Dans cette perspective, la dominance d'un œil doit parvenir à surmonter la rivalité binoculaire.

Une telle hypothèse tend à se confirmer dans une récente étude longitudinale de 214 enfants [59]. Leurs mères ont pu elles-mêmes, étant orthoptistes, observer régulièrement le comportement et l'alignement oculaire, ainsi que le développement qualitatif de la vision de leur enfant durant les premiers mois de la vie. Il en est conclu que « les mésalignements néonataux surviennent dans les deux premiers mois et reflètent usuellement un développement normal du système de vergence ». Ils représentent « des tentatives précoces à la convergence vers des cibles proches ». Il faut attendre deux mois avant de pouvoir invoquer une " ésotropie », c'est-à-dire un strabisme convergent installé. Ainsi, l'émergence d'une vraie latéralité fonctionnelle au niveau des yeux précède largement celle que l'on observe au niveau des mains. L'usage plus fréquent de l'une des mains au pôle de l'action commence seulement à émerger vers huit ou neuf mois, quand le nourrisson parvient à affiner sa préhension en utilisant « la pince pouce-index »[60,61]. Il apparaît donc, en fin de compte, que la dominance oculaire est stabilisée bien plus tôt que la prévalence manuelle. 


\section{Approche expérimentale des rapports latéraux oil-main}

Les expériences ayant eu pour objectif explicite de vérifier les effets de la dominance oculaire sur les performances manuelles sont extrêmement rares. Il y a trois raisons à cela. La première, déjà évoquée, est que la notion d'œil dominant est restée largement occultée en neurosciences. La deuxième est que les constats de terrain cités précédemment ont seulement retenu l'attention des spécialistes de certains sports d'opposition à leur plus haut niveau de pratique. La troisième est que la plupart des travaux expérimentaux dans cette voie ont été rapportés en français. Le bilan des résultats obtenus dans cette intention déclarée sera donc rapide. Les expériences réalisées dans ce domaine reposent en général sur des tâches dites "d'amorçage " où un signal donné avant le signal de réponse contient une information, valide ou non, sur la localisation spatiale du signal qui va suivre. Ainsi, la validité de l'information liée au présignal est manipulée de façon à entretenir l'incertitude tandis que le sujet se prépare à répondre.

\subsection{Expériences fondées sur le temps de réaction (TR)}

Une étude portant sur les TR manuels a permis de comparer dix gauchers et dix droitiers manuels, la moitié de chaque groupe ayant une latéralité œil-main croisée (soit cinq gauchers manuels ayant l'œil gauche dominant [GG], cinq gauchers manuels ayant l'œil droit dominant [DG], cinq droitiers manuels ayant l'œil droit dominant [DD], cinq droitiers manuels ayant l'œil gauche dominant [GD]) $[62,63]$. Les sujets devaient appuyer sur un bouton avec le pouce d'une main dès que s'allumait l'une des quatre diodes électro-luminescentes alignées horizontalement sur un panneau, respectivement à 8 et 24。 à gauche et à droite d'un point central de fixation du regard. Au niveau de ce point central apparaissait d'abord un présignal donnant une information, soit valide (72\% des présignaux), soit neutre $(20 \%)$, soit erronée $(8 \%)$ sur la localisation de la lampe qui devait s'allumer 250 millisecondes après (signal de réponse). Pour tous les sujets, les réponses de la main droite et de la main gauche étaient distribuées en séries alternées. Dans cette condition d'incertitude spatiale, l'analyse des TR n'a pas montré d'effets de la latéralité manuelle des sujets mais, au contraire, des effets significatifs de leur dominance oculaire. Tous présentaient des TR moyens plus courts avec la main contralatérale à l'œil dominant (Fig. 2).

Une autre expérience a été réalisée avec d'autres sujets selon le même protocole, mais en vision monoculaire, en masquant l'un ou l'autre œil [63]. Les sujets étaient dix droitiers manuels, partagés entre cinq cas de dominance oculaire droite et cinq cas de dominance oculaire gauche. Des séries d'essais randomisées permettaient de tester successivement, avec chaque sujet, quatre conditions différentes d'association entre l'un des yeux et l'une des mains. En vision monoculaire, on peut comparer l'hémirétine temporale et l'hémirétine nasale de chaque œil, selon le côté où survient le stimulus. Cette tâche en monocularité s'est avérée très éprouvante sur le plan attentionnel, entraînant un taux élevé d'erreurs pour réagir aux lampes distales (à 24॰). Au contraire, les lampes proximales (à $8^{\circ}$ ) ont donné lieu à d'excellents TR moyens avec un minimum d'erreurs. L'analyse des données obtenues à $8^{\circ}$ a montré une tendance générale à des réactions plus rapides lorsque l'œil testé était associé à la main contralatérale. Cet avantage s'est vérifié chez les droitiers manuels ayant l'œil gauche dominant (sujets GD), lorsqu'ils associaient précisément ces deux pôles préférentiels (canal visuel œil-gauche-main-droite : TR moyen de $260 \mathrm{~ms}$, contre $310 \mathrm{~ms}$ pour les autres canaux d'association œil-main) (Fig. 3).

En outre, l'analyse montre que l'association croisée entre l'œil gauche dominant et la main droite préférentielle suscite des TR différenciés entre les deux hémichamps, mais de fac,on singulière. Cette différence selon l'hémichamp dépend du type d'information qui a précédé le signal, donc de l'incertitude spatiale. Quand le signal apparaît bien dans l'hémichamp annoncé par le 
présignal (information "valide »), c'est du côté de la main de réponse (ici la droite) que la réaction est plus rapide. Quand le signal apparaît dans l'hémichamp opposé (information « erronée »), les TR manuels sont de même niveau quel que soit l'hémichamp où survient le signal. Dans les autres modalités d'association d'un œil et d'une main, les TR sont plus longs et leur distribution latérale diffère (Fig. 3).

Très récemment, d'autres auteurs ont réalisé une étude en recourant de même à la vision monoculaire pour une tâche plus simple que la précédente, mais sous le contrôle d'une IRMf [64]. Il y est apparu que le « colliculus supérieur » réagit plus fortement si la stimulation est reçue par l'hémirétine nasale, par rapport à l'hémirétine temporale. Au contraire, le noyau géniculé latéral et les aires visuelles primaires corticales ne présentent pas cette asymétrie selon le côté du signal visuel. Nous reviendrons sur cette donnée.

\subsection{Expériences de pointer manuel sur des cibles (TR+TM)}

Des expériences demandant aux sujets de répondre en projetant l'une des mains vers une cible sont un peu plus souvent rapportées depuis quelques années. L'une de ces expériences [12] a ainsi pu tester, au-delà des TR, les temps de mouvement (TM) avec la main préférentielle chez une trentaine de jeunes sujets différemment latéralisés. Le protocole ajoutait à l'incertitude spatiale une incertitude temporelle du type «SOA» ${ }^{4}$, c'est-à-dire une variation aléatoire entre 0 à 600 millisecondes du délai séparant le présignal du signal. La cible à atteindre selon les consignes était, soit à gauche, soit à droite, à $12^{\circ}$ du point central où s'allumait le présignal. Celui-ci était accompagné d'une information spatiale aléatoire (valide, erronée ou neutre). L'analyse des données a révélé des TM plus courts avec la main préférentielle chez les sujets ayant une latéralité œil-main croisée (11 GD et quatre DG). Comparés aux autres sujets (18 DD et deux GG), leur avantage est apparu très significatif (Fig. 4). Ce résultat témoigne d'un effet de la dominance oculaire sur la main contralatérale, cette fois en TM, dès lors que cette main est préférentielle.

Ces premiers travaux centrés sur les effets de la dominance oculaire sont restés isolés. Il en est d'autres cependant, dans les revues spécialisées, qui présentent des résultats ne permettant pas d'exclure d'éventuels effets de l'œil dominant des sujets, faute d'avoir introduit ce facteur dans l'analyse des données. Ainsi, l'un de ces travaux compare 20 droitiers et 20 gauchers sur la base des TR réalisés, lors d'une tâche de pointer, face à un dispositif classique de quatre lampes [65]. L'analyse des données montre que les droitiers manuels réagissent plus vite lorsqu'ils mettent en jeu leur main gauche, tandis que les gauchers ne présentent pas de différences significatives entre les deux mains. Les auteurs mettent en cause à la fois l'asymétrie hémisphérique et le transfert interhémisphérique en fonction des exigences spatiales du mouvement. La dominance oculaire des sujets n'est pas évoquée. On peut regretter cette occasion perdue de vérifier, à propos du TR manuel, l'influence de ce biais visuel susceptible de favoriser, dans une telle tâche, la main gauche des droitiers manuels. Le cas des gauchers était aussi susceptible de réserver quelques surprises dans cette voie.

Il n'y a pas lieu de multiplier les exemples, pourtant nombreux, conduisant au même questionnement que ci-dessus. Il faut donc se tourner vers d'autres pistes bibliographiques.

\subsection{Expériences à partir du paradigme de Poffenberger}

La problématique soulevée ici trouve des données plus utiles dans les travaux étudiant le temps de transfert d'une information d'un hémisphère à l'autre par le corps calleux. Ces études reposent sur une tâche classique de " temps de réaction simple (TRS) ». Le signal visuel apparaît soit à 
droite, soit à gauche du point central de fixation du regard et la réaction presse-bouton est effectuée soit de la main droite, soit de la main gauche, par séries alternées. Typiquement, selon le paradigme de Poffenberger (1912), le TRS est plus long pour les réponses dites « croisées » entre le côté d'apparition du signal et celui de la main, par comparaison avec les réponses « non croisées ». Dans le cas « croisé », la relation signal-main est contralatérale, et l'on en déduit que les aires corticales concernées, visuelles et motrices, ne se trouvent pas dans le même hémisphère. Il faut alors que les deux hémisphères communiquent via le corps calleux. Au contraire, dans le cas des réactions « non croisées » (relation signal-main ipsilatérale), les aires concernées se situant dans le même hémisphère, elles s'y associent directement, faisant ainsi l'économie d'un transfert. En soustrayant le TRS « non croisé » du TRS " croisé », on obtient alors, en principe, une différence positive censée représenter le temps de transfert interhémisphérique. Chez des sujets normaux, il se situe, selon les études, entre 2 et $8 \mathrm{~ms}$, pour un TRS total de l'ordre de 120 à $200 \mathrm{~ms}$. Le temps de transfert devient plus long et difficile à interpréter dans les tâches où un présignal entretient une incertitude spatiale et/ou temporelle, et mesurant alors des «TR complexes » (TRC). Chez les sujets privés de corps calleux, le temps de tranfert est de l'ordre de 30 à 45 ms. Il s'effectue alors par la commissure interconnectant les « colliculi supérieurs » [66].

Le paradigme de Poffenberger a suscité de nombreux travaux qui confirment la valeur positive de ces « différences croisé - non-croisé », chez les droitiers manuels, lorsqu'ils réagissent avec leur main droite. Une revue datant d'une quinzaine d'années et portant sur 16 études de ce type a permis d'en dégager quelques aspects marquants [67]. En particulier, le temps de transfert est plus rapide dans un sens que dans l'autre, en fonction de la main chargée de la réponse. Il est plus avantageux quand c'est la main droite de ces droitiers qui réagit à un signal survenant dans l'hémichamp visuel gauche que dans le cas inverse impliquant leur main gauche avec l'hémichamp droit. Cet avantage d'un transfert callosal vers la main droite des droitiers a été souvent attribué à la spécificité de l'hémisphère droit pour la détection du signal visuel et à celle de l'hémisphère gauche pour l'exécution de la réponse manuelle. Cependant, les auteurs de cette revue [67] envisagent d'interpréter plutôt cela comme "une asymétrie de la transmission de l'information visuo-motrice » pouvant être due au corps calleux. Des travaux plus récents dans cette voie montrent plutôt une conduction axonale plus rapide au sein de l'hémisphère droit [68], au stade perceptif et prémoteur [69].

Ainsi, dans cette tâche et avec des droitiers - supposés avoir une latéralité œil-main homogène, les données semblent claires. Lorsque la main droite est impliquée dans la réaction, celle-ci est dite croisée quand le stimulus survient dans l'hémichamp visuel gauche. Ce signal est alors reçu et traité comme information visuelle dans l'hémisphère droit d'où il est activement dirigé vers l'hémisphère gauche, hôte de l'aire motrice manuelle droite. Quand c'est la main gauche qui est impliquée, le croisement inverse est relativement plus coûteux en temps. Ce serait donc l'hémisphère droit qui conditionnerait le mieux le transfert d'un signal visuel vers la main préférentielle des droitiers. Qu'en est-il des gauchers?

Une étude avec le même protocole (Poffenberger) a bien été consacrée à un groupe de gauchers manuels pour le comparer à un groupe témoin de droitiers [70]. Les résultats montrent « des effets de la latéralité manuelle suggérant que les sujets gauchers tendent à avoir des interactions hémisphériques plus efficientes ». S'agit-il alors d'un schéma symétrique de celui des droitiers ? Le processus central est-il toujours conforme au modèle de Poffenberger ? Ce rapport élude, comme les précédents, un rôle éventuel de la dominance oculaire dans les données recueillies. Il se peut, cependant, que cette dominance soit peu sollicitée avec ce protocole. 


\section{Vers une interprétation des processus en jeu}

Rappelons que des enquêtes réalisées dans les sports d'opposition, au plus haut niveau des classements mondiaux, ont révélé une fréquence exceptionnelle des gauchers manuels et surtout, fait inattendu, que ceux-ci présentaient en général une dominance oculaire droite. En outre, de telles « latéralisations œil-main croisées » se sont aussi avérées plus fréquentes chez les droitiers manuels appartenant à cette population de sportifs, par rapport à la population générale. Il restait à comprendre le processus permettant à l'œil dominant de favoriser, dans de tels cas, la main qui lui est contralatérale.

On a pu remarquer dans la section qui précède une sorte de discordance entre les données issues du paradigme de Poffenberger et celles provenant des tâches expérimentales du type « amorçage » citées précédemment pour la comparaison des TR. En effet, à partir du protocole de Poffenberger, on obtient des TR plus courts « quand le signal et la main sont dits "non croisés" » (situés du même côté). Dans une situation expérimentale du type " amorçage »-suggérée par les duels sportifs - les plus courts TR sont obtenus « quand l'œil dominant et la main de réponse sont dits "croisés" » (n'appartenant pas au même côté). Ces résultats ne comparent pas les mêmes choses à l'étage visuel. Ce qui croise ou non, par rapport à la main, ne relève pas du même domaine : dans un cas, c'est le côté où apparaît le signal - et il n'est pas question d'œil dominant - dans l'autre, c'est le siège anatomique de l'œil dominant. Or, nous savons que dans le premier cas, on mesure des TR dits simples, en raison d'une très faible incertitude, tandis que dans le second cas on mesure des TR dits complexes, à cause des conditions d' " amorçage » qui conduisent les sujets à inhiber ou à réorienter certaines réponses.

En réalité, cette discordance entre les résultats fait apparaître qu'il s'agit de deux problématiques différentes. Et il s'y vérifie l'hypothèse ayant précisément conduit à introduire en laboratoire de fortes conditions d'incertitude afin de voir émerger des effets de latéralité tels que ceux provenant de la dominance oculaire $[12,63]$. Ainsi, c'est bien le système visuel qui apparaît sollicité de façon différente dans les deux types de procédures comparées ici.

En l'absence d'incertitude - ou si elle est trop faible - seul semble être requis le sous-système rétino-tectal, le plus automatique. Le processus mis en jeu est alors de type exogène, se déroulant « de bas en haut ». Et ce sont simultanément les hémirétines nasales de l'œil droit et de l'œil gauche qui, couvrant l'ensemble du champ, sont disponibles pour recueillir le signal. Leurs voies centripètes croisent, via le colliculus supérieur, en direction des aires visuelles contralatérales. Chacune des mains étant contrôlée respectivement à partir d'une aire contralatérale, c'est un schéma symétrique qu'illustre le paradigme de Poffenberger.

En situation de forte incertitude, un plus haut niveau d'attention visuelle est requis. Il faut se tenir prêt à corriger in extremis certaines des réponses prévues. C'est l'exemple typique d'un processus endogène se déroulant " de haut en bas », nécessaire pour ajuster les réponses aux conditions aléatoires de survenue du signal. C'est alors le sous-système géniculo-strié qui entre en jeu pour s'emparer du signal détecté et soumettre la réponse aux conditions introduites par le présignal. Nous savons déjà que le noyau géniculé latéral est, en quelque sorte, le « gardebarrière » de l'attention visuelle $[55,56]$. Il est la première étape d'un traitement complexe du signal saisi par l'œil dominant [54]. En conséquence, selon l'œil qu'il relaie sur la voie centripète, le NGL est, d'un côté, le jalon déterminant de la dominance oculaire tandis que, de l'autre côté, il exerce un rôle suppresseur de l'entrée visuelle [57]. Il y a donc une asymétrie fonctionnelle entre les deux NGL, mais ils ne sont pas influencés par l'asymétrie d'hémichamp visuel dépendant du côté d'apparition du signal. Ce biais d'hémichamp est pris en compte auparavant par les « colliculi supérieurs » pour orienter la saccade oculaire. Une étude en IRMf, 
déjà citée ici, a bien montré cela [64]. Au niveau des NGL, c'est celui qui relaie l'œil dominant qui a le privilège de contribuer à une vision médiane, coiffant le méridien vertical.

Il reste à mieux préciser les rapports entre l'œil dominant et cette vision centrée sur le méridien vertical. Curieusement, une dernière étude se référant au paradigme de Poffenberger va nous ouvrir cette voie. Cette étude s'est intéressée, à partir de plusieurs travaux antérieurs, aux sujets ayant des profils de réaction atypiques. Ainsi, certains droitiers manuels, jusqu'à $24 \%$ dans l'un des travaux cités, obtiendraient des « différences croisé - non croisé "négatives" » avec leur main droite, ce qui suggère l'absence de transfert hémisphérique avec cette main quand le signal survient à gauche. L'auteur émet des doutes sur les tests effectués au départ pour sélectionner ces droitiers ${ }^{5}$. À propos de ces cas atypiques, l'auteur évoque en premier lieu, soit une dominance hémisphérique mal établie, soit une dextralité manuelle plus « auto-estimée ou déclarée » que « neurale » [71]. Ne s'agirait-il pas plutôt de cas de dominance oculaire gauche ? Il faudrait pour cela que ce protocole sollicite parfois électivement l'œil dominant. Poursuivant par ailleurs sa quête d'explication, le même auteur se tourne vers des données neuropathologiques et anatomiques qui l'amènent sur une piste mieux étayée [72,73]. Attaché à l'idée que seul « l'hémisphère majeur » peut être à l'origine du transfert hémisphérique le plus rapide, il est conduit en même temps à donner une importance spécifique à la vision maculaire. Celle-ci lui apparaît directement liée au « centre de commande ». Une phrase souligne la voie d'interprétation qu'il retient : « la vision maculaire est la province de l'hémisphère majeur » [72, p. 203]. Dès lors, d'après l'auteur, les faits expérimentaux illustreraient un schéma que l'on peut résumer ainsi : la main qui réalise le plus court temps de réaction est contralatérale à l'hémisphère majeur censé bénéficier de la vision maculaire.

Dans une autre de ses publications sur ce thème, l'auteur complète ce qu'il entend par " vision maculaire ». Il y décrit, sous cette appellation, des processus visuels qui pourraient renvoyer à la dominance oculaire telle que nous l'avons définie ici précédemment, mais qu'il ne nomme jamais. Citons-le : «... Si le transfert callosal implique la vision centrale ou maculaire, c'est qu'elle est discernable par nous comme c'est le cas pour l'ouïe ou le tact (. . .). C'est une vision sans coutures dans les méridiens (. . .). L'évidence suggère que la macula fait partie de l'un ou l'autre hémichamp, dépendant de la latéralité individuelle. . . ». Et il déplore qu'un tel processus central « ne trouve aucune explication cohérente dans les théories rivales en vogue à présent (...) sur la latéralité manuelle » [71, p. 332-333]. On reconnaît dans cette évocation une esquisse du rôle - méconnu une fois de plus - de la dominance oculaire tel qu'il se rapporte au méridien vertical rétinien. Or, c'est l'œil dominant qui s'appuie d'emblée sur ce méridien pour guider les processus de vergence lors des saccades oculaires, favorisant ainsi la fusion centrale des projections binoculaires et faisant de sa propre fovea un repère égocentrique pour la représentation visuelle de l'espace d'action.

Pour tâcher de comprendre comment « la macula », c'est-à-dire la vision fovéale, peut se trouver associée à l'un ou à l'autre hémichamp, selon la latéralité individuelle, il faut à présent se tourner vers une courte série de travaux consacrés à l'anatomie d'une zone de la rétine qui longe son méridien vertical. Les publications à ce propos datent, pour les premières, de plus d'un quart de siècle [74], puis sont restées rares et confidentielles. Récemment complétées, il s'y confirme que la berge interne (nasale) du méridien vertical rétinien présente un singulier « empiètement nasotemporal » [75,76]. Il s'agit d'une bande de 2 à 4॰ longeant le méridien du côté nasal, où l'on observe des cellules ganglionnaires dont les projections afférentes diffèrent de celles qui, dans cette zone, sont typiques de l'hémirétine nasale. Au lieu, comme ces dernières, de croiser la ligne médiane pour rejoindre le « colliculus supérieur », puis l'hémisphère contralatéral, celles qui s'en distinguent empruntent la voie rétino-géniculée, comme celles de l'hémirétine temporale, en direction l'hémisphère ipsilatéral [77]. Cette bande d'empiètement naso-temporal joue un rôle 
dans la perception de la profondeur après fusion des deux hémichamps latéraux de l'espace. La présentation de figures stéréographiques au niveau de cette bande a permis de recueillir des potentiels évoqués en bordure de la lisière qui sépare les aires 17 et $18 \mathrm{du}$ cortex visuel ipsilatéral, où s'inscrit précisément le méridien vertical rétinien [78].

Il est probable que cette bande rétinienne permet d'enrichir la représentation du secteur avoisinant le méridien vertical dans l'hémisphère ipsilatéral à l'œil d'origine. Un travail remarquable réalisé par une équipe américaine apporte de nouveaux et solides arguments dans cette perspective [79]. Grâce à des données obtenues en IRMf, cette équipe fait des constatations qui éclairent bien notre problématique. Dans leurs conclusions, les auteurs rappellent d'abord brièvement qu'à un premier degré, le champ visuel est traité à partir des projections contralatérales qui passent par le colliculus supérieur et sont largement représentées dans le cortex visuel. Puis ils s'attachent tout spécialement à ce qui, au second degré, revient à la voie ipsilatérale (rétinogéniculo- striée) dans la représentation centrale de l'espace. Ils soulignent que cette « représentation visuelle ipsilatérale est un système hautement organisé, topographiquement bien intégré avec d'autres aspects de l'organisation corticale visuelle ». Ils décrivent cette représentation ipsilatérale comme focalisée sur la «couture interhémisphérique » et permettant des communications avec « les plus hautes aires » corticales. Cette « couture » contribuerait ainsi à « la construction d'un percept visuel unitaire » [79, p. 822].

Au total, bien que la notion d'un œil dominant ne soit jamais évoquée dans cette dernière série de travaux, on trouve dans les plus récents d'entre eux des arguments qui convergent vers ce concept à partir des phénomènes constatés. Dès lors, comment se dessine le processus permettant à l'œil dominant d'avantager la main contralatérale lorsque sont attendues des réactions rapides à des signaux, en situation de forte incertitude spatiale et temporelle ? C'est à présent devenu plus clair.

\section{Synthèse}

Le processus en cause repose sur un rapport privilégié, par la voie géniculo-striée, entre l'œil dominant et l'hémisphère ipsilatéral [54-56]. Les informations visuelles rassemblées et transportées par ce canal ont une grande spécificité. Elles constituent un percept unitaire du champ spatial, axé sur le méridien vertical, au service de l'attention visuelle. Les fonctions latéralisées du NGL qui représente, d'un côté l'œil dominant, de l'autre l'œil non dominant, introduisent ainsi leur propre asymétrie au sein même des hémisphères cérébraux. Dès lors, cette asymétrie fonctionnelle d'ordre visuel est à distinguer de l'asymétrie hémisphérique plus générale et classique que reflète la latéralité manuelle. La main prévalante est contrôlée par l'hémisphère contralatéral, tandis que l'œil dominant est représenté dans l'hémisphère ipsilatéral. Ainsi, pour que se rejoignent dans le même hémisphère l'entrée visuelle et la commande motrice manuelle, il faut que l'œil dominant et la main de réponse n'appartiennent pas au même côté. Dans cette seule condition, le chemin suivi par l'influx décisif fait l'économie d'un transfert interhémisphérique (Fig. 5). Cela s'est manifesté en laboratoire par un net avantage en TR, lors de tâches d'amorçage faisant intervenir une forte incertitude spatio-temporelle.

En sport, c'est le même schéma fonctionnel qui s'était illustré initialement. Dans les duels sportifs étudiés $[3,12]$, le classement mondial des compétiteurs suggérait certaines différences individuelles de potentiel dépendant de leur latéralisation œil-main : DD, GD, DG ou GG. Or, ce sont bien les DG qui ont émergé au plus haut niveau et de même, mais dans des disciplines relativement différentes, des GD. Tous ces sports d'opposition sont caractérisés par des situations où règne la plus grande incertitude spatio-temporelle. En laboratoire, les deux groupes de sujets DG et GD ont montré des profils de données assez analogues. Il semble cependant que les gauchers manuels DG tirent surtout leur avantage de la spécialisation visuo-spatiale de 
l'hémisphère droit, tandis que les droitiers manuels GD bénéficient d'un meilleur contrôle visuomoteur de leur main préférentielle grâce à l'hémisphère gauche, dont c'est une fonction dominante. Ces profils de réponse différenciés témoignent d'une relative indépendance entre les quatre formules de latéralité œil-main.

Il est clair que le phénomène révélé par les sports d'opposition n'apparaît que dans des situations où l'on doit réagir à la fois vite et bien à des signaux visuels soumis à une grande incertitude spatiale. Dans ces conditions, c'est le sous-système visuel appelé géniculo-strié qui entre en jeu, au service d'une attention maximale, et l'œil dominant y exerce une fonction spécifique.

\section{Conclusion}

Ce qui est le plus connu de la représentation du champ visuel dans le cortex strié relève surtout de l'archaïque sous-système rétino-tectal, passant par le colliculus supérieur, qui permet de couvrir près de $180^{\circ}$ de l'espace. Chaque hémichamp y est représenté dans l'hémisphère contralatéral. Ce sous-système permet, dans le cadre d'une attention diffuse, de détecter certains signaux en vision périphérique afin de susciter, s'il y a lieu, une attention plus focalisée à partir d'une saccade oculaire. Il fonctionne donc plutôt « de bas en haut».

L'autre sous-système, dit rétino-géniculé, beaucoup plus récent d'un point de vue phylogénétique, ne couvre que $120^{\circ} \mathrm{du}$ champ, soit $60^{\circ}$ pour chaque œil. Il coiffe hiérarchiquement le précédent pour en gérer diversement les données, selon les intentions du sujet et ses actions dans l'espace proche. Il fonctionne donc davantage " de haut en bas ", impliquant plusieurs aires frontales. Il recourt à certains référentiels visuo-spatiaux dépendant des buts à atteindre. L'un de ces référentiels, appelé égocentrique, est ancré sur la fovea de l'œil dominant ${ }^{6}$. Cette dominance oculaire, de nature fonctionnelle, est notamment responsable de la représentation centrale du méridien vertical. Une telle dominance latérale permet au système d'éviter les effets perturbateurs d'une rivalité binoculaire lorsque sont exigés des ajustements visuomoteurs précis et rapides (vergence et saccade oculaires, orientation d'un mouvement de la main, etc.) vers une cible soudainement perçue.

L'œil dominant bénéficie du système rétino-géniculé pour conduire l'attention focalisée et centrer la vision sur des objectifs précis. Grâce à ce système, l'œil dominant est mis en rapport privilégié avec l'hémisphère ipisilatéral, après relais par le noyau géniculé latéral. Ce dernier est à la fois le " garde-barrière de l'attention » et, du côté de l'œil dominant, " l'aiguilleur » de ses effets spécifiques latéralisés. Une telle prévalence latérale suscite parfois, selon l'œil qui domine, un relatif décalage de l'axe du champ vers la droite ou vers la gauche, comme on peut le constater pour la perception du « droit devant» [19]. Il en résulte cependant « un percept cyclopéen unifié ") pouvant être centré sur une cible [80].

Ainsi, dans les conditions de tâche qui nous intéressent ici, l'œil dominant est en rapport fonctionnel, via le noyau géniculé latéral, avec l'hémisphère ipsilatéral [54]. Pour la réponse manuelle, au contraire, la main de réponse est en rapport fonctionnel, via son aire motrice, avec l'hémisphère contralatéral. En conséquence, la liaison fonctionnelle entre l'entrée visuelle et la sortie motrice n'implique qu'un hémisphère dans le cas où l'œil dominant et la main de réponse sont eux-mêmes contralatéraux. Un transfert interhémisphérique n'est donc finalement requis que si cet œil et cette main appartiennent au même côté. Ce transfert étant relativement coûteux en temps, un tel schéma fonctionnel permet d'expliquer à la fois les données expérimentales et les constats de terrain. Cet effet facilitateur de l'œil dominant sur la main contralatérale s'est d'abord manifesté sur la " main de réponse », indépendamment de la préférence manuelle, lorsque les protocoles expérimentaux reposaient sur les seules mesures du «temps de reaction ». Il a fallu requérir des mouvements de projection de la main vers des cibles, avec des exigences de vitesse 
et de précision, pour que les mêmes effets apparaissent en « temps de mouvement » sur la main contralatérale, et « à condition qu'elle soit préférentielle ».

Faut-il déduire de cela que les individus ayant une latéralité homogène - la plupart d'entre nous sont plus malhabiles que les autres ? Certainement pas. En situation de découverte visuelle et surtout quand rien ne presse, il est probable que l'implication simultanée des deux hémisphères dans l'exploration visuelle apporte de nombreux avantages. Dans certains sports également, comme le tir à l'arc ou à la carabine, chez un droitier homogène, l'hémisphère droit est au service du repérage spatial de la cible, tandis que l'hémisphère gauche est alors disponible pour gérer la posture et le déclenchement du tir.

Comparativement, une latéralisation œil-main croisée, en particulier dans le cas œil-droit/main gauche, permet d'initier plus vite une réaction de la main gauche en direction d'un signal-cible visuel. Il en résulte un ajustement plus rapide de la réponse. L'économie d'un transfert interhémisphérique permet, dans ces situations, un gain de temps de l'ordre de quelques dizaines de millisecondes. Cet avantage n'est déterminant qu'à courte distance, quand se réduit à l'extrême le temps de décision, comme c'est la règle en sport d'opposition. N'oublions pas, cependant, qu'une coopération des deux hémisphères ne tarde pas à s'instaurer à partir de la réaction initiale, ne serait-ce que pour mieux assurer l'exécution de la réponse.

Le modèle ci-dessus permet d'expliquer l'effet de l'œil dominant sur la main préférentielle, tel que l'ont révélé les typologies individuelles s'illustrant dans les compétitions sportives. Notons cependant que certaines expériences fondées sur le seul temps de réaction ont accordé un avantage à la main contralatérale indépendamment de la prévalence manuelle. Cela suggère que la main contralatérale à l'œil dominant bénéficie d'un potentiel avantageux pour des tâches visuo-manuelles avec incertitude spatiale.

Plusieurs observations de terrain tendent à confirmer cela. Il s'est avéré qu'un entraînement spécifique de la main contralatérale à l'œil dominant, même lorsqu'elle n'était pas a priori la main préférentielle, permet de tirer avantage du schéma fonctionnel évoqué ci-dessus. On en connaît plusieurs exemples en sport de haut niveau [12]. L'un des plus célèbres est celui du champion le plus titré de l'histoire de l'escrime (23 titres mondiaux ou olympiques à l'épée et au fleuret). Étant en réalité droitier homogène œil-main-pied, il a commencé la pratique de l'escrime avec sa main droite à huit ans, puis son père, maître d'armes, lui a appris à 12 ans à se servir de sa main gauche. Dès lors, c'est sous l'étiquette acquise d'un gaucher manuel ayant l'œil droit dominant qu'il a longtemps brillé en compétition aux armes d'estoc.

Au total, la dominance d'un œil apparaît comme un trait psychomoteur individuel comparable à celui qui se manifeste au niveau des membres par la prévalence d'une main ou d'un pied.

Cependant, l'œil dominant se distingue du cas de la main par quelques traits singuliers :

- sa plus forte composante perceptive, surtout spatiale ;

- sa plus fréquente variante senestre (25 à 30\% des individus) ;

- son rapport ipsilatéral avec l'hémisphère recevant ses projections.

Les données rassemblées dans cette revue semblent annoncer de prochaines avancées scientifiques concernant les effets fonctionnels de la dominance oculaire.

\section{Références}

[1] Landers DM. Moving competitive shooting into the scientist's lab. Am Rifle 1980;128:36-8. [2] Azémar G, Ripoll H, Simonet P, Stein JF. Etude neuropsychologique du comportement des gauchers en escrime. Cinésiologie 1983;22:7-18.

[3] Azémar G. Les gauchers en escrime : données statistiques et interprétation. Escrime Int 1993;7:15-9. 
[4] Carson RG, Chua R, Elliott D, Goodman. The contribution of vision to asymmetries in manual aiming. Neuropsychologia 1990;28(11):1215-20.

[5] Barthelemy S, Boulinguez P. Orienting visuospatial attention generates manual reaction time asymmetries in target detection and pointing. Behav Brain Res 2002;133(1):109-16.

[6] Stuphorn V, Bauswein E, Hoffmann KP. Neurons in the primate superior colliculus coding for arms movements in gaze-related coordinates. J Neurophysiol 2000;83(3):1283-99.

[7] Rizzolatti G, Rigglo L, Dascola X, Umiltà C. Reorienting attention across the horizontal and vertical meridians: evidence in favor of a premotor theory of attention. Neuropsychologia 1987;25(1A):31-40.

[8] Elliott D, Lyons J, Chua R, Goodman D, Carson RG. The influence of target perturbation on manual aiming asymmetries in right-handers. Cortex 1995;31(4):685-97.

[9] Boulinguez P, NougierV,Velay JL. Manual asymmetries in reaching movements control, I: Study of right-handers. Cortex 2001;37(1):101-22.

[10] Pisella L, Grea H, Tilikete C, Vighetto A, Desmurget M, Rode G, et al. An "automatic pilot" for the hand in human posterior parietal cortex: toward reinterpreting optic ataxia. Nat Neurosci 2000;3(7):729-36.

[11] Van Donkelaar P, Lee JH, Drew AS. Transcranial magnetic stimulation disrupts eye-hand interactions in the posterior parietal cortex. J Neurophysiol 2000;84(3):1677-80.

[12] Azémar G. L’Homme Asymétrique : Gauchers et droitiers face-à-face. Paris: CNRSEditions; 2003, 315 p.

[13] Della Porta GB. De refractione. Optics Parte. Libri Novem. Ex Officina Horatij Salvania. Naples: Apud Io Iacobum Carlinum and Anotinium Pacem, 1593.

[14] Tong F, Meng M, Blake R. Neural bases of binocular rivalry. Trends Cogn Sci 2006;10(11):502-11.

[15] Lankheet MJM. Unraveling adaptation and mutual inhibition in perceptual rivalry. J Vis 2006;6:304-10.

[16] Hibbard PB. A statistic model of binocular rivalry. Vis Cognit 2007;15(2):149-65.

[17] Oishi A, Tobimatsu S, Arakara K, Taniwaki T, Kira J. Ocular dominance in conjugate movements at reading distance. Neurosci Res 2005;52(3):263-8.

[18] Perennou DA, Amblard D, Laassel el-M, Pelissier J. Hemispheric asymmetry in the visual contribution to postural control in healthy adults. Neuroreport 1997;8(14):3137-41.

[19] Porac C, Coren S. Sighting dominance and egocentric localization. Vis Res 1986;26(10):1700-13.

[20] Paillard J. L'encodage sensori-moteur et cognitif de l'expérience spatiale. In: Paillard J, editor. La lecture sensori-motrice et cognitive de l'expérience spatiale : Directions et Distances. Paris: Éditions du CNRS, Comportements; 1980. p. 217-25.

[21] Berthoz A. Le sens du mouvement. Paris: Odile Jacob; 1997, 345 p.

[22] Muller JR, Philiastides MG, Newsome WT. Microstimulation of the superior colliculus focuses attention without moving the eyes. Proc Natl Acad Sci U S A 2005;102(3):521-3.

[23] Carlsen AN, Chua R, Inglis JT, Sanderson DJ, Franka IM. Can prepared responses be stored subcortically? Exp Brain Res 2004;159(3):301-9.

[24] Snyder LH, Calton JL, Dickinson AR, Lawrence BM. Eye-hand coordination: saccades are faster when accompagned by a coordinated arm movement. J Neurophysiol 2002;87(5):2279-86.

[25] Mennie N, Hayhoe M, Sullivan B. Look-ahead fixations: anticipatory eye movements in natural tasks. Exp Brain Res 2007;179(3):427-42.

[26] Hanes DP, Wurtz RH. Interaction of the frontal eye field and superior colliculus for saccade generation. J Neurophysiol 2001;85(2):804-15. 
[27] Pierrot-Deseilligny C, Muri RM, Ploner CJ, Gaymard B, Rivaud-Pechoux S. Cortical control of ocular saccades in humans: a model for motricity. Prog Brain Res 2003;142:3-17.

[28] Hopp JJ, Fuchs AF. Investigating the site of human saccadic adaptation with express and targeting saccades. Exp Brain Res 2002;144:538-48.

[29] Isa T, Saito Y. The direct visuo-motor pathway in mammalian superior colliculus: novel perspective on the interlaminar connection. Neurosci Res 2001;41(2):107-13.

[30] Isa T, Kobayashi Y. Switching between cortical and subcortical sensorimotor pathways. Prog Brain Res 2004;143:299-305.

[31] May PJ, Hartwich-Young R, Nelson J, Sparks DL, Porter JD. Cerebellotectal pathways in the macaque: implications for collicular generation of saccades. Neurosciences 1990;36(2):30524.

[32] Harter MR, Aine C, Schroeder C. Hemispheric differences in the neural processing of stimulus location and type: Effects of selective attention on visual evoked potentials. Neuropsychologia 1982;20:421-38.

[33] Palmer T, Tzeng OJL. Cerebral asymmetry in visual attention. Brain and Cogn 1990;13:4658.

[34] Tzeng OJL, Wang W. Search for common neurocognitive mechanism for language and movements. Am J Psychol Gen 1984;114(3):285-310.

[35] Serrien DJ, Ivry RB, Swinnen SP. Dynamics of hemispheric specialization and integration in the context of motor control. Nat Rev Neurosci 2006;7(2):160-6.

[36] Goodale MA, Milner AD. Separate pathways for perception and action. Trends Neurosci 1992;15(1):20-5.

[37] Valyear KF, Culham JC, Sharif N, Westwood D, Goodale MA. A double dissociation between sensisivity to changes in object identity and object orientation in the ventral and dorsal streams: a human fMRI study. Neuropsychologia 2006;44(2):218-28.

[38] Himmelbach M, Karnath HO. Dorsal and ventral stream interaction: contributions from optic ataxia. J Cogn Neurosci 2005;17(4):632-40.

[39] Hugdahl K. Lateralization of cognitive processes in the brain. Acta Psycholgica 2000;105:211-35.

[40] Patel GA, Sathian K. Visual search: bottom-up or top-down ? Front Biosci 2002;1(5D):16993.

[41] Kapoula Z, Yang Q, Coubard O, Daunys G, Orssaud C. Role of the posterior parietal cortex in the initiation of saccades and vergence: right/left functional asymmetry. Ann NY Acad Sci 2005;1039:184-97.

[42] Buschman TJ, Miller ER. Top-down versus bottom-up control of attention in the prefrontal and posterior parietal cortices. Science 2007;315(5820):1860-2.

[43] Hahn B, Ross TJ, Stein EA. Neuroanatomical dissociation between bottomup and top-down processes of visuospatial selective attention. Neuroimage 2006;15(32):842-53.

[44] Seyal M, Sato S, White BG, Porter RJ. Visual evoked potentiel and eye dominance. Electroencephalogr Clin Neurophysiol 1981;52(5):424-8.

[45] Rombouts SA, Barkhof F, Sprenger M, Valk J, Scheltens P. The functional basis of ocular dominance: functional MRI (fMRI) findings. Neurosci Lett 1996;221(1):1-4.

[46] Ooi TL, Optom B, He ZJ. Sensory eye dominance. Optometry 2001;72(3):168-78.

[47] Valera EM, HellerW, Berenbaum H. A twin study of individual differences in perceptual asymmetry. Laterality 1999;4(3):299-311.

[48] Han Y, Seideman M, Lennerstrand G. Dynamics of accommodative vergence movements controlled by the dominant and non-dominant eye. Acta Ophtalmol Scand 1995;73(4):319-24. 
[49] Zhou Y, Yu H, Yang Y, Shou T. Non-dominant eye responses in the dorsal lateral geniculate nucleus of the cat: an intracellular study. Brain Res 2003;987(1):76-85.

[50] Handa T, Mukuno K, Uozato H, Niida T, Shoji N, Shimizu K. Effects of dominant and nondominant eyes in binocular rivalry. Optom Vis Sci 2004;81(5):377-83.

[51] Handa $T$, Uozato $H$, Higa R, Nitta M, Kawamorita $T$, Ishikawa $H$, et al. Quantitative measurement of ocular dominance using binocular rivalry induced by retinimeters. J Cataract Refract Surg 2006;32(5):831-6.

[52] Azémar G. Bon pied, bon oil : méthode de détermination d'un indice de latéralisation fonctionnelle. In: Podologie 96. Paris: Expansion Scientifique franc, aise; 1996, 7-14.

[53] Seijas O, Liano PG, Liano RG, Roberts CJ, Piedrahita E, Diaz E. Ocular dominance diagnosis and its influence in monovision. Ann J Ophtalmol 2007;144(2):209-16.

[54] Haynes JD, Deichmann R, Rees G. Eye-specific effects of binocular rivalry in the human lateral geniculate nucleus. Nature 2007;438(7067):496-9.

[55] Wunderlich K, Schneider KA, Kastner S. Neural correlates of binocular rivalry in the human lateral geniculate nucleus. Nat Neurosci 2005;8(11):1595-602.

[56] Kastner S, Pinsk MA. Visual attention as a multilevel selection process. Cogn Affect Behav Neurosci 2004;4(4):483-500.

[57] Haynes JD, Deichmann R, Rees G. Eye-specific suppression in human LGN reflects perceptual dominance during binocular rivlary. Nature 2005;438(7067):496-9.

[58] Shneor E, Hochstein S. Eye dominance effects in feature search. Vision Res 2006; 46(25): 4258-69.

[59] Horwood A. Neonatal ocular misalignments reflect vergence development but rarement become esotropia. Br J Ophtalmol 2003;87(9):1146-50.

[60] Azémar G. La préhension chez des enfants de 7 à 9 mois. In: La motricité de la naissance à six ans. Paris: INSEP; 1972, 53-54.

[61] Morange-Majoux F, Peze A, Bloch H. Organization of left and right hand movement in a prehension task: a longitudinal study from 20 to 32 weeks. Laterality 2000;5(4):351-62.

[62] Azémar G, Ripoll H, Stein J-F, Nougier V. Lefthanders and sport: ocular laterality effect on distribution of attention. Paper presented at the Scientific Congress of the Olympic Games, Séoul, jul. 1988.

[63] Azémar G. Asymétries fonctionnelles et performances visuo-motrices. In: Ripoll et $\mathrm{H}$, Azémar G, editors. Neurosciences du Sport. Paris: INSEP; 1987. p. 228-42.

[64] Sylvester R, Josephs O, Driver J, Rees G. Visual fMRI responses in human superior colliculus show a temporal-nasal asymmetry that is absent in lateral geniculate and visual cortex. J Neurophysiol 2007;97(2):1495-502.

[65] Velay JL, Daffaure V, Raphael N, Benoit-Dubrocard S. Hemispheric asymmetry and interhemispheric transfer in pointing depend on the spatial components of the movement. Cortex 2001;37(1):75-90.

[66] Savazzi S, Fabri H, Rubboli G, Paggi A, Tassinari CA, Marzi CA. Interhemispheric transfer following callosotomy in humans: Role of the superior colliculus. Neuropsychologia 2007;45(11):2417-27.

[67] Marzi CA, Bisiacchi P, Nicoletti R. Is interhemispheric transfer of visuo-motor information asymmetric ? Evidence from a meta-analysis. Neuropsychologia 1991;29(12):1163-77.

[68] Barnett KJ, Corballis MC. Speeded right-to-left information transfer: the result of speeded transmission in right-hemisphere axons ? Neurosci Lett 2005;380(1-2):88-92.

[69] Cavina-Pratesi C, Bricolo E, Pelligrini B, Marzi CA. At what stage of manual visual reaction time does interhemispheric transmission occu: controlled or ballistic ? Exp Brain Res 2004;155(2):220-30. 
[70] Cherbuin N, Brinkman C. Hemispheric interactions are different in lefthanders individuals. Neuropsychology 2006;20(6):700-7.

[71] Derakhshan I. Laterality of seizure onset and the simple reaction time: revamping the Poffenberger's paradigm for seizure surgery. Neurol Res 2006;28(7):777-84.

[72] Derakhshan I. Handedness and macular vision: laterality of motor control underpins both. Neurological Res 2004;26:331-7.

[73] Derekhshan I. Crossed-uncrossed differnce (CUD) in a new light: anatomy of the negative CUD in Poffenberger paradigm. Acta Neurol Scand 2006;113(3):203-8.

[74] Perenin MT, Vadot E. Macular sparing investigated by means of Haidinger brushes. Br J Ophtalmology 1981;65:429-35.

[75] Leventhal AG, Ault SJ, Vitek DJ. The nasotemporal division in primate retina: the bases of macular sparing and splittng. Science 1988;240(4848):66-7.

[76] Reinhard J, Trauzetell-Klosinski S. Nasotemporal overlap of retinal ganglion cells in humans: a functional study. Invest Ophtalmol Vis Sci 2003;44(4):1568-72.

[77] Fukuda Y, Sawai H, Watanabe M, Wakakuwa K, Morikawa K. Nasotemporal overlap of crossed and uncrossed retina ganglion cell projections in japanese monkey (macaca fuscata). J Neurosci 1988;2: 2353-73.

[78] Nakajima S, Nikara T. VEPs induced by binocular disparity stimulus of the retinal nasotemporal overlap. Nippon Ganka Gakkai Zasski 1994;98(3):298-305.

[79] Tootell RBH, Mendola JD, Hadjikhani NK, Liu AK, Dale AM. The representation of ipsilateral visual field in human cerebral cortex. Proc Natl Acad Sci U S A 1998;95:818-24.

[80] Liversedge SP, Rayner K, White SJ, Findlay JM, McSorley E. Binocular coordination of the eyes during reading. Curr Biol 2006;16(17): 1726-9.

\section{Notes}

1 Il n'est pas suffisant d'attribuer la réussite des gauchers à leur rareté par rapport aux droitiers car on compte presque autant de gauchers que de droitiers dans l'élite du fleuret et de l'épée.

2 Dans la presse scientifique internationale, on appelle ainsi « œil dominant » celui qui guide les mouvements coordonnés des deux yeux. Cependant, en pratique ophtalmolgique, on l'appelle plutôt « œil directeur » et l'on réserve le qualificatif « dominant » à la fonction sensorielle.

3 SOA: stimulus onset asynchrony.

4 Test hold-in-card : Le sujet doit être debout devant une table, tenant des deux mains, à bout de bras, une feuille de bristol percée en son milieu d'un trou large d'un centimètre. Il lui est d'abord demandé de maintenir cet orifice tout contre un texte posé sur la table et de commencer à lire par ce moyen. Il doit ensuite s'arrêter sur un mot de ce texte, puis rapprocher la feuille de son visage, lentement, de façon à maintenir son regard sur le même mot. Lorsque la feuille est près du visage, le sujet est à même de constater - parfois avec surprise - qu'un seul œil a poursuivi cette tâche. C'est l'œil dominant qui s'est chargé de maintenir la fixation du regard sur l'objectif lors d'un passage progressif de la binocularité à une inévitable monocularité. Ce test, qui met bien en jeu la rivalité binoculaire pour en faire émerger l'œil dominant, s'avère constant en pratique répétée.

5 Il n'est pas rare de découvrir des travaux où l'on s'est seulement référé à « la main qui écrit ». 
6 Il s'agit ici essentiellement de tâches où intervient en premier la fonction visuelle. Dans des tâches sportives sollicitant surtout la proprioception et la fonction vestibulaire (acrobatie, gymnastique, lutte) ce référentiel égocentrique renvoie plutôt au centre de gravité du corps entier. En effet, " le cerveau n'utilise pas un seul, mais de multiples référentiels en fonction de la tâche à accomplir et des indices sensoriels utilisables ou essentiels. » (Berthoz A [10]). 
Fig. 1. Schéma de l'organisation des voies centripètes du système visuel.

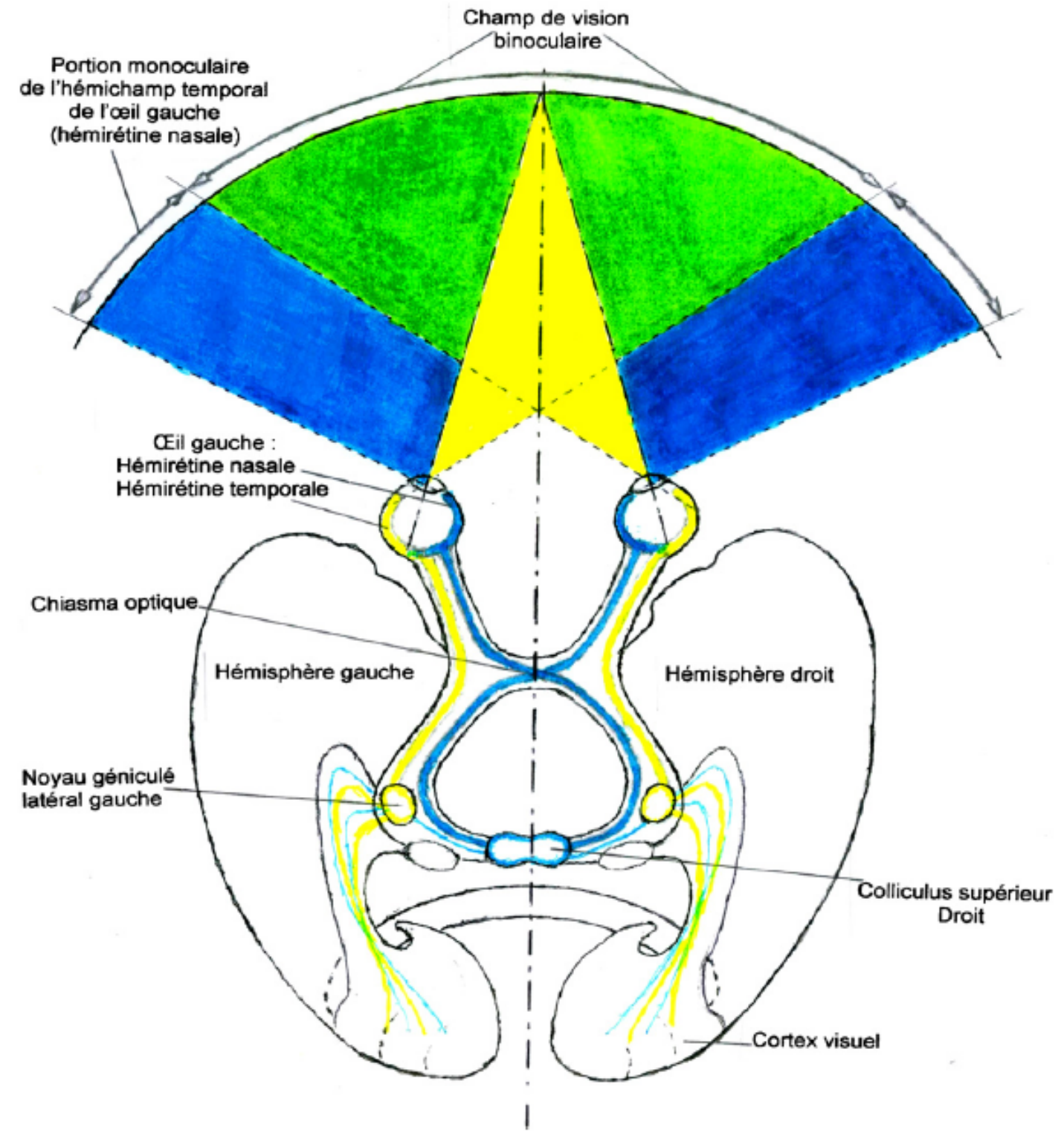


Fig. 2. Comparaison des temps de réaction entre main droite et main gauche en fonction de la dominance oculaire individuelle. Chez tous les sujets, les TR sont plus courts avec leur main contralatérale à l'œil dominant, indépendamment de leur latéralité manuelle (p < 0,01).

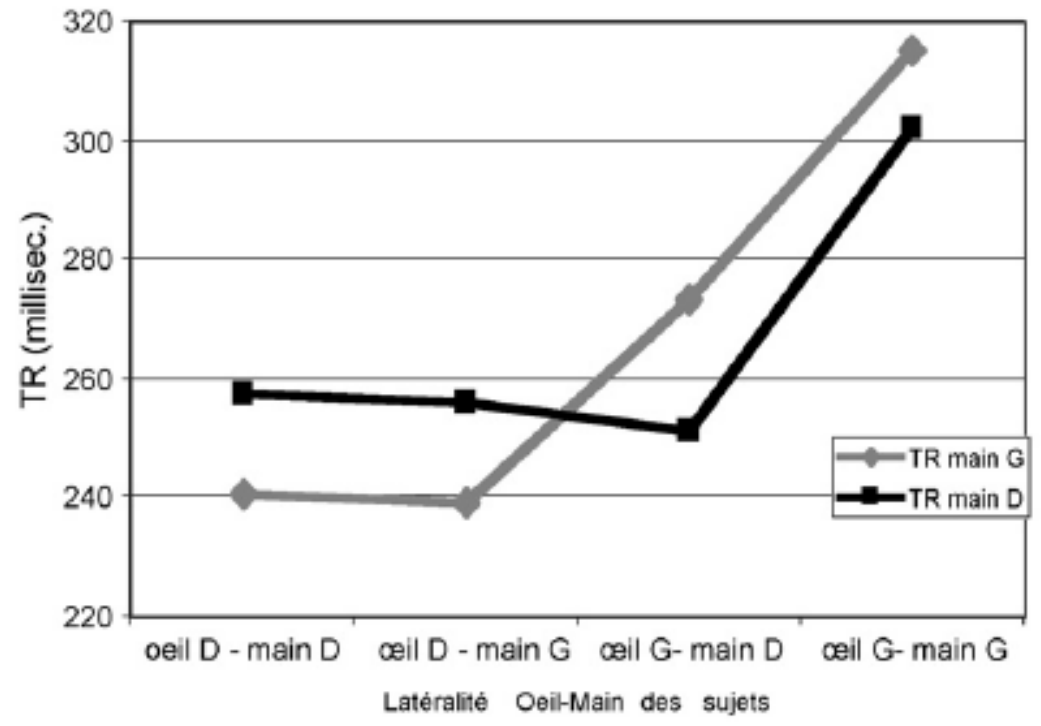


Fig. 3. Expérience en monocularité. Comparaison des TR de la main droite de droitiers manuels en fonction de leur dominance oculaire : on observe les meilleures performances chez ceux qui ont une dominance oculaire gauche lorsqu'ils sont testés en vision monoculaire gauche (3b : canal œilG/main D).

(a) Droitiers manuels ayant

l'oeil droit dominant (réponse main droite)

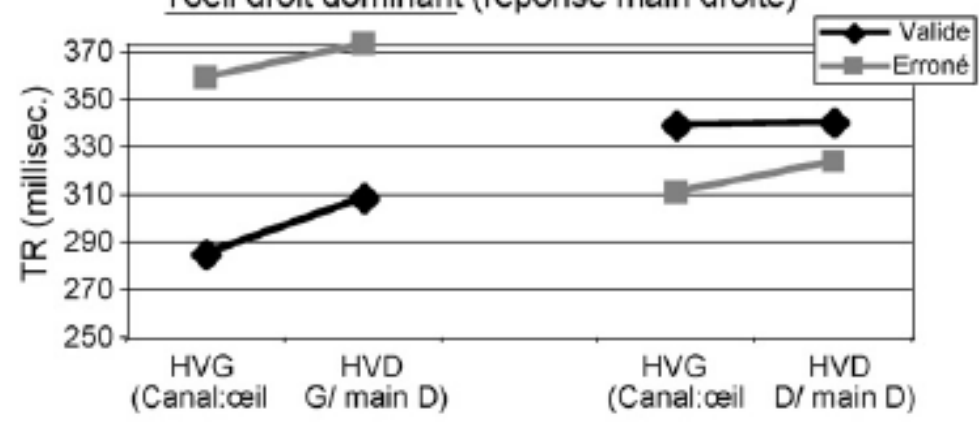

(b) Droitiers manuels ayant l'oeil gauche dominant (réponse main droite)

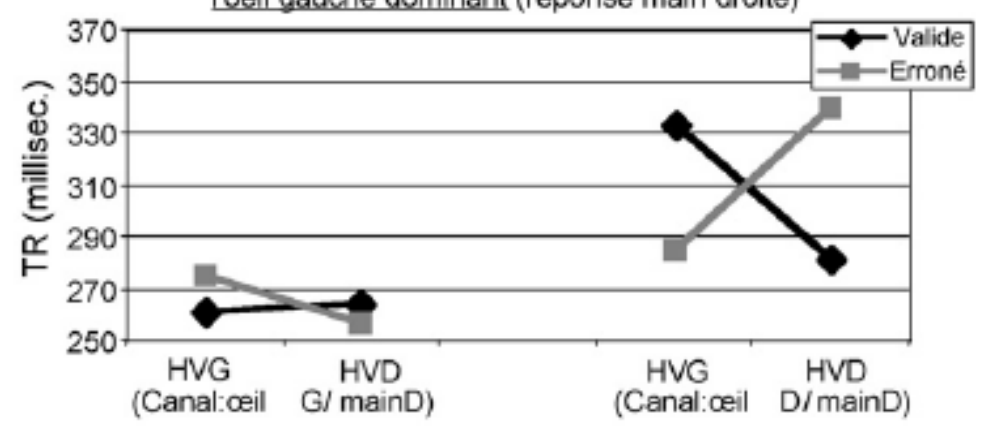


Fig. 4. Tâche de projection de la main préférentielle (go-nogo). Comparaison des TM moyens entre droitiers et gauchers manuels, en fonction de leur dominance oculaire. Dans les deux groupes, les plus rapides ont une latéralité œil-main contralatérale $(p<0,001)$.

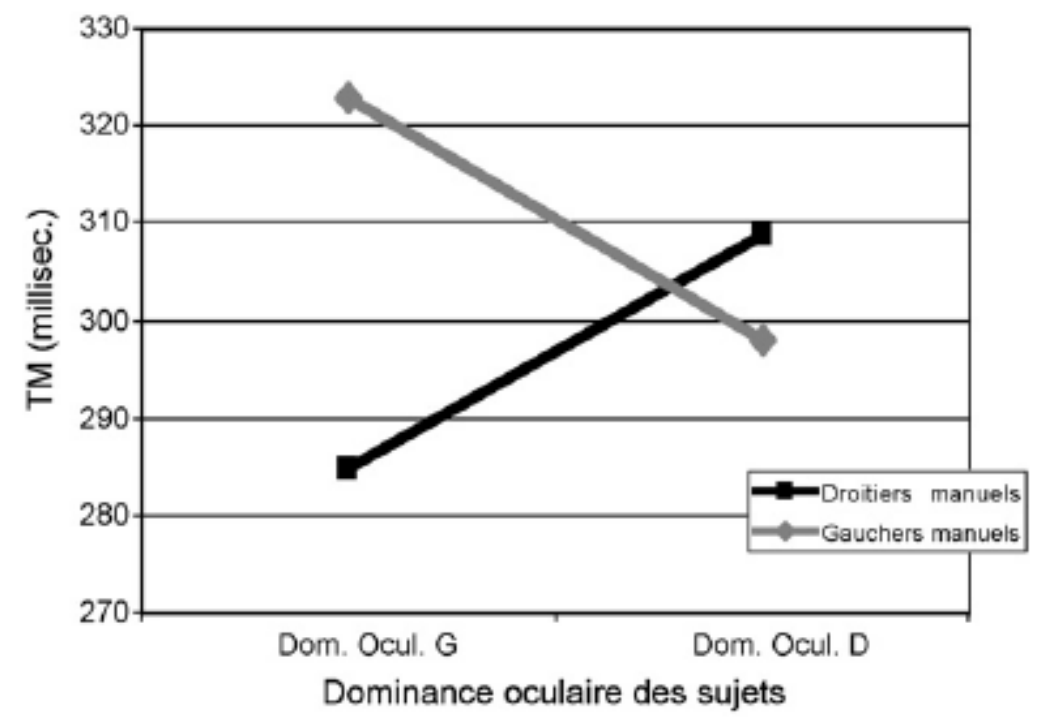


Fig. 5. Représentation schématique du circuit visuomoteur mis en jeu entre l'œil et la main, en situation d'incertitude spatiale, dans le cas d'un gaucher manuel ayant une dominance oculaire droite.

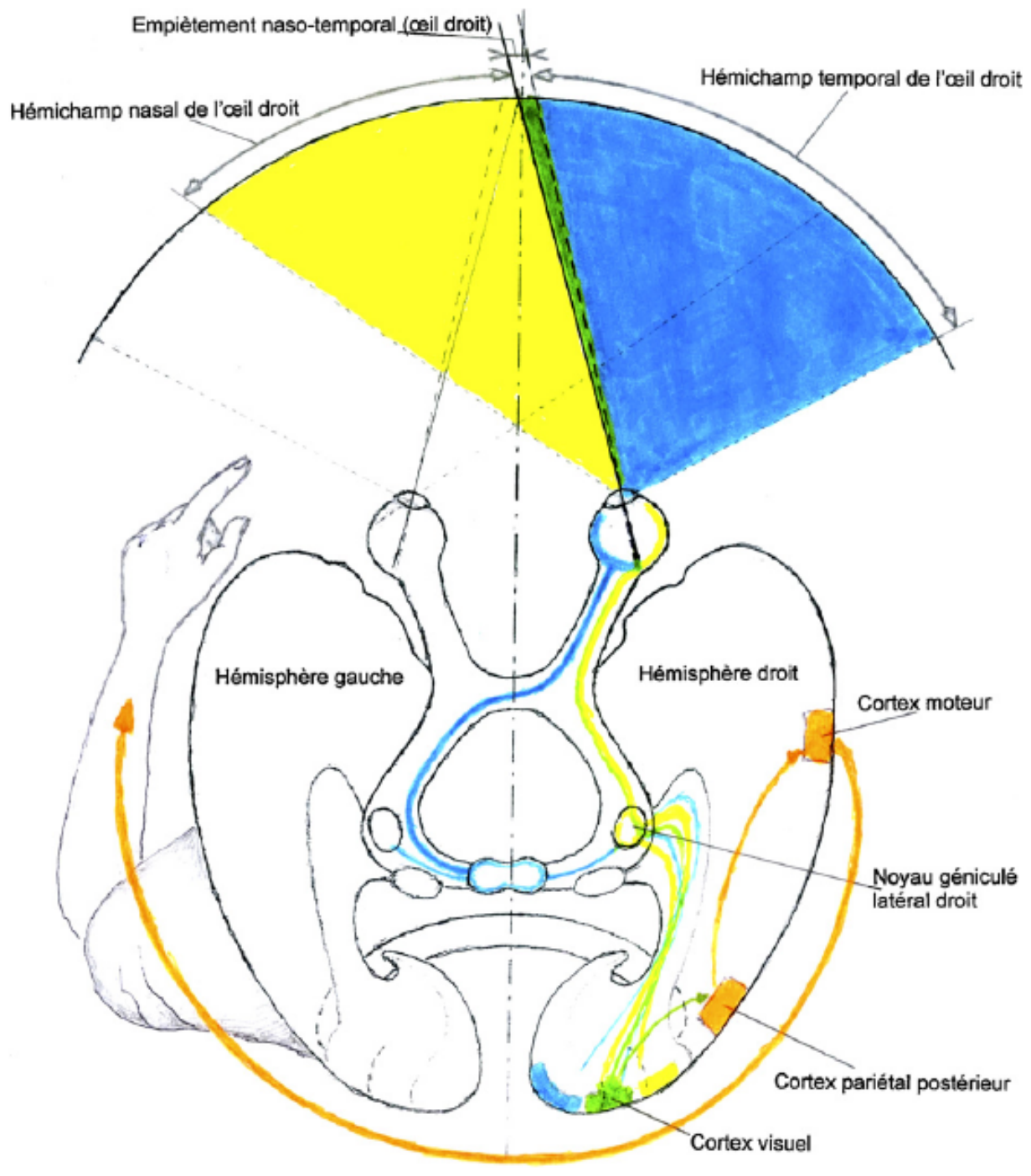

